\title{
Assessment of Carotid Intima Media Thickness and Left Ventricular Mass Index in Children with Idiopathic Nephrotic Syndrome
}

\author{
Heba Mostafa Ahmed (iD) \\ Emad El-Deen Ameen' \\ Mohammad Shafiq Awad ${ }^{2}$ \\ Osama Ezzat Botrous' \\ 'Department of Pediatrics, Faculty of \\ Medicine, Beni-Suef University, Beni Suef, \\ Egypt; ${ }^{2}$ Department of Cardiology, \\ Faculty of Medicine, Beni- Suef University, \\ Beni Suef, Egypt
}

\begin{abstract}
Background: Children with nephrotic syndrome (NS) are at a greater risk of atherosclerosis due to recurrent exposures to hyperlipidemia, hypertension, and immunosuppressive medications. CIMT (carotid intima media thickness) is a reliable marker for assessment of atherosclerosis of large and medium-sized blood vessels; endothelial dysfunction and increased CIMT usually precede the development of cardiovascular diseases. Some manifestations of NS, like proteinuria and hyperlipidemia, are associated with an increased risk of cardiac morbidity and mortality. The aim of the current study was to evaluate the carotid intima media thickness and LVM (left ventricular mass) thickness in children with nephrotic syndrome.
\end{abstract}

Subjects and Methods: Eighty-one children with nephrotic syndrome and 100 healthy children as controls were enrolled in the study. The inclusion criteria were: disease duration of minimum of 12 months, glomerular filtration rate $>60 \mathrm{~mL} / \mathrm{min} / 1.73 \mathrm{~m}^{2}$ and children aged two years or more at the time of study. CIMT and left ventricular mass index, lipid profile, protein/creatinine ratio in urine and kidney function tests were done for cases and controls after approval of internal ethical committee.

Results: The mean CIMT (mm) was significantly higher in NS $(0.51 \pm 0.12)$ compared to controls $(0.42 \pm 0.09)(\mathrm{P}<0.001)$. LVM and LVM Index were significantly higher in NS than controls ( $\mathrm{p}<0.001$, for both). Subsequently, CIMT was significantly correlated to duration of the disease $(p<0.001)$, LVM index was significantly correlated with duration of the disease, body mass index (BMI), blood pressures and triglycerides level $(\mathrm{p}<0.05)$.

Conclusion: Children with NS are at increasing risk to develop atherosclerosis as measured by CIMT. LVM was significantly higher in NS and positively correlated to BP, disease duration, triglyceride levels and BMI.

Keywords: nephrotic syndrome, children, left ventricular hypertrophy, carotid intima media thickness, dyslipidemia

\section{Introduction}

Nephrotic syndrome (NS) is considered one of the commonest types of renal disorders in children. ${ }^{1}$ The clinical features of NS include massive proteinuria, hypoalbuminemia, as well as generalized edema resulting from destruction of the glomerular basement membrane. ${ }^{2}$ Hyperlipidemia is also a usual feature found in patients with NS. ${ }^{1}$ Proteinuria was reported as a remarkable risk factor for cardiovascular disorders. Several metabolic abnormalities such as hypercholesterolemia, hypertriglyceridemia, and hypercoagulable states are related to the severity of proteinuria and contribute to the development of cardiovascular diseases. Other
Correspondence: Heba Mostafa Ahmed Department of Pediatrics, Faculty of Medicine, Beni- Suef University, Beni-Suef, Egypt

Tel +20I00I5I664I

Email heba_most@yahoo.com 
factors as hypertension, renal insufficiency, and corticosteroids treatment may also contribute to the development of cardiovascular diseases(CVD). ${ }^{3}$ Despite hyperlipidemia is often intermittent and temporary in steroid-sensitive nephrotic syndrome (SSNS); yet, dyslipidemia along with other abnormalities might be persistent in patients suffering frequently relapsing (FRNS) or steroid-resistant (SRNS) nephrotic syndrome. ${ }^{4}$ Ultrasound assessment of the carotid intima media thickness (CIMT) was used as a marker of atherosclerosis of the coronary arteries. Increased CIMT and plaque formation in the carotid arteries are remarkable indicators for cardiovascular diseases in the general population. ${ }^{5}$ The aim of this study was to evaluate CIMT and left ventricular mass (LVM) in children with idiopathic nephrotic syndrome.

\section{Methodology}

This study was carried out in the period from April 2019 till August 2020. Eighty-one children with idiopathic NS following up at the pediatric-nephrology outpatient clinic were included in the study. Patients were categorized according to their response to steroid treatment into steroid-sensitive (SSNS), steroid-dependent (SDNS), and steroid-resistant (SRNS). One-hundred age and sex-matched healthy children selected from the general outpatient clinic were included in the study as a control group. The inclusion criteria were: age more than two years at the time of enrollment in the study, continuous or interrupted treatment for a minimum of 12 months before enrollment, glomerular filtration rate more than $60 \mathrm{~mL} / \mathrm{min} / 1.73 \mathrm{~m} 2$, being free of acute infection in the previous three months before the study. The exclusion criteria were: family history of early atherosclerosis, cerebrovascular accidents or familial hyperlipidemia, patients receiving lipid-lowering agents, systemic vasculitis in activity, coexisting cardiac anomalies, children with chronic kidney disease, presence of severe edema at time of examination, congenital or rheumatic heart disease, and high CRP at the time of the study. All patients were subjected to detailed history taken including past medical and drug history, clinical data of nephrotic patients stressing on age at diagnosis, duration of the disease, response to steroid treatment, frequency of relapses, immunosuppressive medications, and full clinical assessment including measurement of weight, height, body mass index (BMI), arterial blood pressure (BP), examination for edema and cardiac examination to ensure none of our patients had congenital or rheumatic heart disease. Laboratory investigations including levels of blood urea, creatinine, albumin, spot protein/creatinine in urine (PCR), serum $\mathrm{Na}$, serum $\mathrm{K}$, and blood urea. All children underwent a complete lipid profile consisting of total cholesterol, high-density lipoproteins-cholesterol (HDL), lowdensity lipoproteins-cholesterol (LDL), and triglycerides (TG). Cholesterol and TG were measured through 2 steps: 1) the use of the enzymatic endpoint method to precipitate LDL using phosphotungstic acid/magnesium followed by the removal of HDL. 2) LDL cholesterol was calculated by Friedwald's formula

$$
\begin{aligned}
& \mathrm{LDL} \text { cholesterol } \mathrm{mg} / \mathrm{dl}=\{\text { Total cholesterol } \\
&-\mathrm{HDL} \text { cholesterol }- \text { plasma triglyceride } / 5\}
\end{aligned}
$$

\section{CIMT Measurement}

Measurement of CIMT was performed according to Mannheim's Consensus Guideline $2011^{6}$ by an experienced cardiologist who was blinded to the patient's status. The ultrasound measurement was obtained using a single ultrasound machine (VIVID SS) with a linear high-frequency transducer (7.5 MHz). The patients were lying in the supine position and had head turned slightly to the contralateral side with slight overextension of the neck. After resting for ten minutes, to obtain the arterial diameter measurements, the arterial wall segment was assessed in the longitudinal view, firmly at a right angle to the ultrasound beam, with both walls obviously seen. It is made of two parallel lines representing the two structural boundaries: the intima lining the lumen and mediaadventitia. Measurements were obtained from the far wall of the left common carotid artery $10-20 \mathrm{~mm}$ proximal to the bifurcation. Images of the thickest CIMT were taken and measurements were taken on it while the calipers positioned on a zoomed common carotid artery image. Images were attained at passive end-expiration to reduce overall cardiac movement from the standard parasternal long-axis and apical planes. The standard views were used to obtain the left ventricle (LV) dimensions. Modified Simpson's method was used to calculate the left ventricle end-systolic and end-diastolic volumes and then they were used to calculate the ejection fraction. The LVM index was calculated using the Devereux's formula by the following equation: Left Ventricular Mass (LVM) $=0.80[1.04 \times($ interventricular septal thickness + posterior wall thickness + end-diastolic diameter) 3 - (enddiastolic diameter) 3] +0.6 . LVMI is calculated as LVM/surface area. 


\section{Study Definitions}

Relapse: 24 hours urinary proteins more than $40 \mathrm{mg} / \mathrm{m} 2 / \mathrm{h}$ or urinary proteins more than $3+$ by dipstick in spot sample for at least three consecutive days.

Remission: 24 hours' urinary proteins less than $4 \mathrm{mg} / \mathrm{m} 2 / \mathrm{h}$, nil or trace by dipstick for at least three consecutive days

Frequent relapses: Two or more relapses within 6 months of steroid-induced remission; 4 or more relapses in any 12 months.

Steroid dependence: at least 2 consecutive relapses during treatment cortisone with or within 2 weeks of its withdrawal.

Steroid resistance: Failure to achieve remission after 4-8 weeks of daily oral prednisolone therapy at a dose of $2 \mathrm{mg} / \mathrm{kg} /$ day. $^{7}$

\section{Statistical Analysis}

The SPSS software (Statistical Package for the Social Sciences, version 22.0, SPSS Inc, Chicago, IL, USA) was used for analysis. Comparison between qualitative data was carried out using the chi-square and values were expressed as numbers and percentages. Parametric quantitative independent groups were examined using the $t$-test or the Mann-Whitney test and values were expressed as mean \pm standard deviation. One way Anova test was used to compare continuous variables between the three disease groups. Correlations between the study's continuous variables were done using the Pearson correlation coefficient test. General linear multivariate analysis was done to confirm significant correlations between risk factors and both CIMT and LVM index. P-value was set at less than 0.05 for significant results.

\section{Results}

This study included 81 children with nephrotic syndrome and 100 age and sex-matched healthy children as controls. The included children were stratified according to their age into 3 groups: G1; $\leq 5$ years (19 patients and 37 controls), G2; 6-10 years (33 patients, 38 controls), and $\mathrm{G} 3$; >10 years (29 patients and 25 controls). The age at disease onset in the patients was $4.9 \pm 2.6$ (range 1.2-12 years). In the patients' group, 31 (38.3\%) patients were steroid-sensitive nephrotic syndrome (SSNS), 36 (44.4\%) were steroid-dependent nephrotic syndrome (SDNS) and14 (17.3\%) were steroid-resistant nephrotic syndrome (SRNS). The mean number of relapses among patients was $2.1 \pm 1.9$ (range $0-7$ ) and mean disease duration of
$3.6 \pm 2.6$ (range $1-11$ years). Among patients with SDNS and SRNS, 35 patients had done renal biopsy; 28 patients had minimal change disease (MCD), 6 patients had focal segmental glomerulosclerosis (FSGS) and one patient had mesangial-proliferative glomerulonephritis (MesPGN). Among our patients, $42 \%$ of them had received immunosuppressive therapy rather than steroids. $19.7 \%$ of our patients received cyclophosphamide, $24.7 \%$ received calcineurin inhibitors (CNI), 6.2\% received mycophenolate mofetil $(\mathrm{MMF})$ and 6\% received combined therapy $(\mathrm{CNI}+\mathrm{MMF})$. The clinical and laboratory characteristics of the study groups are shown in (Table $1)$. Ten of our patients $(12.3 \%)$ were hypertensive at the time of study enrollment and 17 patients (21\%) showed hypertriglyceridemia versus $4(5 \%)$ children in the control group $(\mathrm{p}=0.023)$ and was positively correlated to systolic blood pressure $(\mathrm{r}=0.38, \mathrm{p}=0.021)$. On comparing the two study groups regarding the CIMT and LV parameters, all were significantly higher in patients as compared to controls across the three age groups (Table 2). On stratification of the patients according to their response to steroids, patients with SRNS had significantly higher blood pressures, PCR, and TG levels as compared to SSNS patients. LVM was significantly higher in SRNS. On the contrary, there were no significant differences between the three groups as regarding other echo findings (Table 3 ). In comparing patients with MCD and FDGS according to renal biopsy there were no significant differences between the two groups regarding demographic, clinical, and laboratory data except for PCR $(0.346 \pm 0.59$ versus $3.75 \pm 7.84 \mathrm{mg} / \mathrm{gm}$ in $\mathrm{MCD}$ and FSGS, respectively, $\mathrm{p}=0.024)$. On the other hand, the LVM index was significantly higher in the FSGS group as compared to MCD (Table 4). Correlations between the echocardiographic data and other clinical and laboratory data are shown in (Table 5). CMIT was significantly correlated to disease duration, while there were no significant correlations with systolic blood pressure, diastolic blood pressure, or lipid profile. LVM was significantly correlated to disease duration, BMI, systolic and diastolic blood pressures, TG, and HDL cholesterol level. The results of multivariate analysis had confirmed these associations (Table 6).

\section{Discussion}

Endothelial dysfunction is an initial reversible phase in the pathogenesis of atherosclerosis and can predict cardiovascular diseases. CIMT is an indirect indicator of 
Table I Clinical and Investigational Data of Cases and Controls

\begin{tabular}{|c|c|c|c|c|}
\hline & & Cases (Mean \pm SD) & Controls (Mean \pm SD) & $\mathbf{p}$ \\
\hline \multicolumn{2}{|c|}{ Age (years) } & $8.80 \pm 3.44$ & $7.65 \pm 3.66$ & 0.103 \\
\hline \multicolumn{2}{|c|}{ Weight (kg) } & $30.71 \pm 10.63$ & $26.28 \pm|| .7 \mid$ & 0.04 \\
\hline \multicolumn{2}{|c|}{ Height $(\mathrm{cm})$} & $121.60 \pm 21.88$ & $129.43 \pm 19.54$ & 0.041 \\
\hline \multicolumn{2}{|c|}{ BMI $\left(\mathrm{kg} / \mathrm{m}^{2}\right)$} & $17.55 \pm 3.18$ & $16.26 \pm 1.46$ & 0.013 \\
\hline \multicolumn{2}{|c|}{ Systolic BP (mmHg) } & $109.07 \pm 9.12$ & $104.37 \pm 10.81$ & 0.01 \\
\hline \multicolumn{2}{|c|}{ Diastolic BP (mmHg) } & $70.88 \pm 6.17$ & $68.00 \pm 6.07$ & 0.01 \\
\hline \multicolumn{2}{|c|}{ Urea (mg/dl) } & $23.42 \pm 7.18$ & $20.35 \pm 3.56$ & 0.012 \\
\hline \multicolumn{2}{|c|}{ Creatinine $(\mathrm{mg} / \mathrm{dl}$} & $0.42 \pm 0.12$ & $0.4 I \pm 0.13$ & 0.737 \\
\hline \multicolumn{2}{|c|}{$\mathrm{Na}(\mathrm{mE} / \mathrm{L}$} & $139.27 \pm 2.54$ & $|40.17 \pm 2.5|$ & 0.068 \\
\hline \multicolumn{2}{|c|}{$\mathrm{K}(\mathrm{mE} / \mathrm{L})$} & $6.10 \pm 16.75$ & $4.27 \pm 0.30$ & 0.492 \\
\hline \multicolumn{2}{|c|}{$\mathrm{PCR}(\mathrm{mg} / \mathrm{mg}$ creatinine) } & $0.63 \pm 2.21$ & $0.09 \pm .03$ & 0.039 \\
\hline \multicolumn{2}{|c|}{ TG (mg/dl) } & $139.81 \pm 219.99$ & $105.72 \pm 38.53$ & 0.334 \\
\hline \multicolumn{2}{|c|}{ HDL-cholesterol(mg/dl) } & $54.98 \pm 9.39$ & $66.52 \pm 13.48$ & $<0.001$ \\
\hline \multicolumn{2}{|c|}{ LDL-cholesterol (mg/dl) } & $119.37 \pm 93.67$ & $81.24 \pm 28.74$ & 0.014 \\
\hline \multicolumn{2}{|c|}{ Cholesterol (mg/dl) } & $205.20 \pm 112.54$ & $148.77 \pm 26.49$ & 0.001 \\
\hline \multirow[t]{2}{*}{ Sex } & Female & 27 (33.3\%) & $30(30.0 \%)$ & 0.79 \\
\hline & Male & $54(66.7 \%)$ & $70(70.0 \%)$ & \\
\hline \multicolumn{2}{|c|}{ Albumin (gm/dl) } & $3.6 \pm .69$ & $4.825 \pm 1.02$ & 0.02 \\
\hline \multicolumn{2}{|l|}{$\mathrm{EF}$} & $72.01 \pm 8.37$ & $68.32 \pm 4.19$ & 0.010 \\
\hline \multicolumn{2}{|c|}{ CIMT (mm) } & $0.5 I \pm 0.12$ & $0.42 \pm 0.09$ & $<0.001$ \\
\hline \multicolumn{2}{|c|}{ LVM (gm) } & $70.38 \pm 24.59$ & $44.85 \pm 11.96$ & $<0.001$ \\
\hline \multicolumn{2}{|c|}{ LVM Index $\left(g / m^{2}\right)$} & $67.86 \pm 20.10$ & $49.20 \pm 15.87$ & $<0.001$ \\
\hline
\end{tabular}

Abbreviations: BMI, body mass index; BP, blood pressure; CIMT, carotid intima media thickness; EF, ejection fraction; HDL, high-density lipoprotein; K, potassium; LDL, low-density lipoprotein; LVM, left ventricular mass; Na, sodium; PCR, protein/creatinine in urine; TG, triglycerides.

Table 2 Carotid Intima Media Thickness, LVM and LVM Index According to Age Groups in Cases and Controls

\begin{tabular}{|c|c|c|c|c|}
\hline & Age Group & Cases & Controls & $\mathbf{p}$ \\
\hline \multirow[t]{3}{*}{ CIMT } & GI & $0.531 \pm 0.111$ & $0.453 \pm 0.083$ & 0.003 \\
\hline & $\mathrm{G} 2$ & $0.506 \pm 0.117$ & $0.393 \pm 0.07988$ & $<0.001$ \\
\hline & G3 & $0.52 I \pm 0.090$ & $0.442 \pm 0.113$ & 0.005 \\
\hline \multirow[t]{3}{*}{ LVM } & GI & $62.926 \pm 12.296$ & $53.466 \pm 13.512$ & 0.013 \\
\hline & $\mathrm{G} 2$ & $75.746 \pm 15.357$ & $64.636 \pm 20.827$ & 0.013 \\
\hline & G3 & $82.892 \pm 19.335$ & $64.101 \pm 22.643$ & 0.002 \\
\hline \multirow[t]{3}{*}{ LVM index } & GI & $86.200 \pm 19.498$ & $66.947 \pm 33.971$ & 0.026 \\
\hline & G2 & $79.256 \pm 15.062$ & $68.187 \pm 20.88 \mid$ & 0.014 \\
\hline & G3 & $76.344 \pm 18.802$ & $57.500 \pm 16.05 \mid$ & $<0.001$ \\
\hline
\end{tabular}

Abbreviations: CIMT, carotid intima media thickness; LVM, left ventricular mass.

atherosclerosis and targeted organ destruction in adults. Its implication in children is still under estimation however there are growing numbers of studies on children with risk factors for vascular injury. ${ }^{8}$ Impaired endothelial function was reported in the active phase of idiopathic nephrotic syndrome. Previous studies had reported significant correlations between CIMT and disease duration and response to treatment in children with NS. ${ }^{9}$ This study was designed to investigate and assess the CIMT and left ventricular mass in idiopathic NS. There was a significant increase in weight and BMI in NS patients compared to controls. Similar results were reported by other studies. ${ }^{10-12}$ In the study by Youssef et al, ${ }^{13}$ they reported that body weight and BMI were significantly higher in NS patients than controls $(p<0.001)$. This finding can be explained by the excess weight gain during steroid therapy which can persist even after termination of steroids and usually associated with dyslipidemia that can increase the 
Table 3 Clinical and Investigational Data of the Three Nephrotic Groups

\begin{tabular}{|c|c|c|c|c|}
\hline & SSNS $(n=30)($ Mean \pm SD $)$ & SDNS $(n=36)($ Mean \pm SD $)$ & SRNS $(n=15)($ Mean \pm SD $)$ & $\mathbf{p}$ \\
\hline Age (years) & $7.60 \pm 2.99$ & $9.50 \pm 3.34$ & $9.53 \pm 4.03$ & 0.05 \\
\hline Age at onset (years) & $4.68 \pm 1.96$ & $4.84 \pm 2.98$ & $5.60 \pm 2.68$ & 0.52 \\
\hline Disease duration(years) & $2.98 \pm 2.43$ & $4.14 \pm 2.55$ & $3.56 \pm 2.73$ & 0.186 \\
\hline Weight (kg) & $25.76 \pm 10.14$ & $31.32 \pm 9.44$ & $32.16 \pm 12.82$ & 0.05 \\
\hline Height $(\mathrm{cm})$ & $121.06 \pm 17.18$ & $133.80 \pm 18.29$ & $130.26 \pm 23.27$ & 0.02 \\
\hline BMI $\left(\mathrm{kg} / \mathrm{m}^{2}\right)$ & $16.60 \pm 4.62$ & $16.97 \pm 1.55$ & $18.14 \pm 2.39$ & 0.31 \\
\hline Systolic BP (mmHg) & $99.83 \pm 8.14$ & $105.83 \pm 8.66$ & $108.33 \pm 9.19$ & 0.003 \\
\hline Diastolic BP (mmHg) & $65.66 \pm 5.04$ & $70.27 \pm 6.20$ & $72.00 \pm 5.60$ & 0.001 \\
\hline Urea $(\mathrm{mg} / \mathrm{dl})$ & $21.85 \pm 6.32$ & $22.73 \pm 5.87$ & $28.20 \pm 9.75$ & 0.013 \\
\hline Creatinine $(\mathrm{mg} / \mathrm{dl})$ & $0.38 \pm 0.09$ & $0.42 \pm 0.10$ & $0.20 \pm 0.05$ & 0.066 \\
\hline $\mathrm{Na}(\mathrm{mE} / \mathrm{L}$ & $138.56 \pm 2.67$ & $139.47 \pm 2.24$ & $140.20 \pm 2.80$ & 0.104 \\
\hline $\mathrm{K}(\mathrm{mE} / \mathrm{L})$ & $9.20 \pm 27.53$ & $4.26 \pm 0.27$ & $4.30 \pm .40$ & 0.446 \\
\hline PCR (mg/mg creatinine) & $0.151 \pm 0.059$ & $0.18 \pm 0.09$ & $1.93 \pm 4.98$ & 0.018 \\
\hline TG (mg/dl) & $106.80 \pm 46.39$ & $109.38 \pm 98.09$ & $278.80 \pm 471.83$ & 0.023 \\
\hline HDL-cholesterol(mg/dl) & $54.14 \pm 4.99$ & $56.30 \pm 9.57$ & $53.53 \pm 14.67$ & 0.526 \\
\hline LDL-cholesterol (mg/dl) & $110.83 \pm 85.96$ & $107.11 \pm 76.25$ & $165.12 \pm 131.84$ & 0.127 \\
\hline Cholesterol (mg/dl) & $185.06 \pm 88.44$ & $174.38 \pm 86.50$ & $250.80 \pm 156.63$ & 0.055 \\
\hline Albumin $(\mathrm{gm} / \mathrm{dl})$ & $3.90 \pm 0.92$ & $3.65 \pm 0.1 .02$ & $3.2 \pm 1.11$ & 0.03 \\
\hline EF & $69.36 \pm 6.17$ & $72.91 \pm 8.75$ & $75.20 \pm 10.15$ & 0.060 \\
\hline CIMT (mm) & $0.5 I \pm 0.10$ & $0.5 I \pm 0.13$ & $0.5 I \pm 0.12$ & 0.964 \\
\hline LVM (gm) & $61.90 \pm 20.10$ & $66.16 \pm 25.43$ & $82.06 \pm 25.42$ & 0.043 \\
\hline LVM Index $\left(g / \mathrm{m}^{2}\right)$ & $64.50 \pm 17.12$ & $63.25 \pm 15.67$ & $79.86 \pm 41.18$ & 0.046 \\
\hline
\end{tabular}

Abbreviations: BMI, body mass index; BP, blood pressure; CIMT, carotid intima media thickness; EF, ejection fraction; HDL, high-density lipoprotein; K, potassium; LDL, low-density lipoprotein; LVM, left ventricular mass; Na, sodium; PCR, protein/creatinine in urine; SDNS, steroid dependent NS; SRNS, steroid resistant NS; SSNS, steroid sensitive nephrotic syndrome; TG, triglycerides.

Table 4 Comparison Between Patients with MCD $(n=28)$ and FSGS $(n=6)$ as Regard CIMD and Echo Findings

\begin{tabular}{|l|c|c|c|}
\hline & FSGS & MCD & p \\
\hline EF & $73.00 \pm 6.03$ & $75.11 \pm 1.61$ & 0.644 \\
CIMT $(\mathrm{mm})$ & $0.51 \pm .13$ & $0.50 \pm .14$ & 00.843 \\
$\operatorname{LVM}(\mathrm{gm})$ & $75.25 \pm 30.62$ & $71.00 \pm 18.54$ & 0.747 \\
$\operatorname{LVMI}\left(\mathrm{gm} / \mathrm{m}^{2}\right)$ & $64.74 \pm 22.74$ & $91.83 \pm 43.27$ & 0.037 \\
\hline
\end{tabular}

Abbreviations: CIMT, carotid intima media thickness; EF, ejection fraction; FSGS, focal segmental glomerulosclerosis; LVM, left ventricular mass; MCD, minimal change disease.

cardiovascular risk. ${ }^{14}$ We also noted that height was significantly lower in patients than controls, similar findings were reported by Candan et al. ${ }^{15}$ This finding can be explained by the negative effect of steroids on linear growth through the suppression of osteoblastogenesis and the induction of osteocyte and osteoblast apoptosis which lead to a decrease in bone formation. ${ }^{16}$ Serum cholesterol, LDL cholesterol, and protein/creatinine in urine were significantly higher in patients than controls while serum albumin and HDL cholesterol were significantly lower in patients compared to controls. Similar results were reported by other studies. ${ }^{9,13}$ Patients with NS have
Table 5 Correlations Between the Echocardiography Findings and Other Clinical and Laboratory Data. Values are Expressed as $\mathrm{p}(\mathrm{r})$

\begin{tabular}{|l|l|l|l|}
\hline & CIMT & LVM & LVM Index \\
\hline Disease duration & $<0.00$ I (0.6I3) & $<0.00$ I (0.454) & 0.0 II (0.23I) \\
BMI & $0.870(-0.015)$ & $<0.00 I(0.363)$ & $0.0 I(0.3 \mathrm{I})$ \\
Systolic BP & $0.084(0.158)$ & $0.003(0.266)$ & $0.038(0.273)$ \\
Diastolic BP & $0.073(0.147)$ & $0.006(0.250)$ & $0.016(0.244)$ \\
PCR & $0.725(0.040)$ & $0.98(0.002)$ & $0.65(0.050)$ \\
TG & $0.059(0.172)$ & $0.002(0.283)$ & $0.035(0.284)$ \\
HDL-cholesterol & $0.530(-0.058)$ & $0.014(-0.222-)$ & $0.033(-0.194-)$ \\
LDL-cholesterol & $0.528(0.059)$ & $0.13(0.143)$ & $0.53(0.059)$ \\
Cholesterol & $0.636(0.043)$ & $0.39(0.079)$ & $0.88(0.014)$ \\
\hline
\end{tabular}

Abbreviations: BMI, body mass index; BP, blood pressure; CIMT, carotid intima media thickness; HDL; high-density lipoprotein; LDL, low-density lipoprotein; LVM, left ventricular mass; PCR, protein/creatinine in urine; TG, triglycerides.

a higher risk for myocardial infarction and coronary vascular accidents compared to normal populations. Hyperlipidemia, hypoalbuminemia, and hypercoagulable states are relative risk factors in particular LDL cholesterol which could be assumed to increase the oxidative stress through the synthesis of atherogenic factors. ${ }^{17,18}$ CIMT is a marker for the evaluation of atherosclerosis of large and medium-sized blood vessels secondary to risk 
Table 6 General Linear Multivariate Model for Analyzing the Effect of Risk Factors on CIMT and LVM Index

\begin{tabular}{|l|l|l|l|l|l|l|}
\hline Dependent Variable & Source & Sum of Squares & df & Mean Square & F & Sig. \\
\hline CIMT & Nephrotic & 0.150 & 5 & 0.150 & 11.009 \\
& Disease duration & 213.627 & 5 & 42.725 & 0.001 \\
& Disease duration & 350.259 & 43 & 8.146 & 10.239 \\
& BMI & 148.120 & 43 & 37.030 & 1.709 & 0.046 \\
& SYSTOLIC BP & 4131.389 & 43 & 96.079 & 5.631 & 1.408 \\
& DIASTOLIC BP & 2090.833 & 43 & 48.624 & 0.045 \\
& TG & $3,626,515.923$ & 43 & $84,337.580$ & 1.876 & 12.719 \\
& HDL & 6443.353 & 43 & 103.925 & $<.027$ \\
& & & 3.000 & 0.000 \\
\hline
\end{tabular}

Abbreviations: BMI, body mass index; BP, blood pressure; CIMT, carotid intima media thickness; HDL; high-density lipoprotein; LVM, left ventricular mass; TG, triglycerides.

factors as hypertension, hyperlipidemia, and endothelial dysfunction. ${ }^{19}$ In the current study, CIMT was significantly higher in children with NS in comparison to controls across the three age groups especially in the age group 6-10 years. Similar results were documented in the study by Hooman et $\mathrm{al}^{20}$ who declared that common carotid artery CIMT in the patients group $(0.42 \pm .14)$ was higher than that of the control group $(0.37 \pm .08, \mathrm{p}<0.05)$. Correspondingly, Mehta et $\mathrm{al}^{21}$ in their study conducted on 66 children with NS and 128 age and sex matched healthy controls, found that CIMT was significantly higher in NS especially in the age above 4 years. However, unlike our results, Kniazewska et $\mathrm{al}^{9}$ found no significant differences in CIMT between 30 children previously treated for nephrotic syndrome versus 30 healthy children. In this study, the inclusion in the study required being in remission free of steroids for at least 4 years in contrary to our study which included children with SDNS and SRNS on current therapy with steroids or other immunotherapies. We did not find a significant difference in the CIMT between different steroid response groups or children with MCD and FSGS. Similar findings were reported by Rahul et $\mathrm{a}^{12}$ who found no significant difference in CIMT between patients with IFRNS, FRNS, SDNS, and SRNS but they did not study the difference between patients according to their histopathology. Also, Youssef et $\mathrm{al}^{13}$ did not state a difference between response subgroups or patients in remission and activity as regards the CIMT. CIMT was significantly correlated to disease duration. Similar to our results, Hooman et $\mathrm{al}^{20}$ found that CIMT was correlated with disease duration longer than 2 years $(\mathrm{P}$ $=0.04,95 \%$ confidence interval, 0.002 to 0.12 ). Even after excluding children with a glomerular filtration rate less than $90 \mathrm{~mL} / \mathrm{min} / 1.73 \mathrm{~m} 2$, this correlation remained significant $(\mathrm{P}=0.03)$. We had not found any correlation of CIMT with blood pressure, and this could be because only $12.2 \%$ of our NS children were hypertensive. Similar to our results, Mehta et $\mathrm{al}^{21}$ reported no significant correlation between CIMT and blood pressure. Moreover, there were no significant correlations between CIMT and lipid profile in our patients. Similar findings were reported by other studies. ${ }^{13,20,22}$ In our study, LVM and LVMI were significantly higher in the NS group than controls. Also, LVM index was significantly higher in SRNS and in patients with FSGS compared to MCD. Similar to our study, Candan et $\mathrm{al}^{15}$ found that LVM index values were higher in NS than healthy children in their study conducted on 37 NS patients to assess the presence of understated cardiovascular disease (CVD) in children with SRNS. In our study, there were significant positive correlations between LVM index and disease duration, BMI, systolic BP, and TG and on multivariate analysis disease duration, BMI, BP and TG were independent risk factors for LVH. In the study by Candan et $a l^{15}$ the only independent risk factor for LVH was BMI and they failed to elicit significant correlation between LVM index and other laboratory and clinical parameters. The limitation of their study was the small number of patients, which may alter the rationality of the multivariate analysis. Systemic hypertension is a considerable risk factor for the development of renal injury and cardiovascular disease and abnormal BP was formerly noted in patients with chronic kidney disease. $^{23}$ In our study, both systolic and diastolic blood pressures were significantly higher in patients than controls and in SRNS than SSNS and were positively correlated to LVM index. In the study by Frishberg et $\mathrm{al}^{24}$ on 30 children with SRNS, they documented that the analysis of consecutive echocardiograms of their patients revealed 
mild to severe LVH at the onset of SRNS and reported an additional increase in LVH during follow up, parallel with the acquirement of substantial hypertension. Similarly, Hooman et $\mathrm{al}^{20}$ reported that echocardiographic measurements showed LVH in one $(1.9 \%)$ of the control group versus $19(50 \%)$ of the NS group ( $p$ value $<0.001$ ) and there was a positive correlation between LVH and systolic hypertension ( $p$-value $=0.019$ ). Despite there was no significant difference between patients and controls as regards the mean TG levels but the frequency of hyperlipidemia was significantly higher in nephrotic patients and it was significantly correlated to both blood pressure and LVMI indicating the role of dyslipidemia as a risk factor for hypertension and LVH. In our study, the HDL-cholesterol was significantly lower in patients than controls and there was a significant inverse correlation between LVMI and HDL-cholesterol. The same finding was reported by Giuseppe et $\mathrm{al}^{25}$ signifying that HDL-cholesterol may have a protecting role against LVH. In addition to the induction of reverse cholesterol transport, HDL also has antioxidant activities and anti-inflammatory properties and protects against endothelial dysfunction by binding to scavenger receptor $\mathrm{B} 1$ to activate endothelial nitric oxide synthase. $^{26}$ During NS, there is dysregulation of several key proteins resulting in abnormal structure and function of HDL-cholesterol as well as deficiency of lecithin cholesterol acyltransferase due to its loss in urine. ${ }^{27}$

Limitations of our study were lack of long-term follow up to see the changes in CIMT over a period of time and lack of studying the effect of corticosteroids cumulative dose on CIMT.

\section{Conclusions}

CIMT was increased in children with NS and there was significant positive correlation with disease duration. Similarly, LVM index was higher in cases than controls and was significantly positively correlated with BMI, $\mathrm{BP}$, disease duration and TG levels. Children with NS are at more risk for atherosclerosis as measured by CIMT and LVH as measured by LVM index compared to healthy children. Therefore, follow-up and early management of risk factors such as proper control of hypertension to maintain the blood pressure on the $50 \%$ for age and sex, control of dyslipidemia by healthy nutrition and proper control of the nephrotic state and proteinuria are strongly recommended for these patients.

\section{Ethics Approval and Consent to Participate}

This study was conducted in accordance with the Declaration of Helsinki. It was approved by the Ethics Committee of Beni-Suef University, Faculty of medicine and the Ethic Code was FMBSUREC/05032019. FWA00015574. The objectives of this study were explained for all parents by the researcher and informed consent was obtained from the parents before initiation.

\section{Acknowledgment}

The authors would like to thank all of the children who participated in the study and their parents.

\section{Author Contributions}

All authors made a significant contribution to the work reported, whether that is in the conception, study design, execution, acquisition of data, analysis and interpretation, or in all these areas; took part in drafting, revising or critically reviewing the article; gave final approval of the version to be published; have agreed on the journal to which the article has been submitted; and agree to be accountable for all aspects of the work.

\section{Disclosure}

The authors report no conflicts of interest in this work.

\section{References}

1. Safaei A, Maleknejad S. Spectrum of childhood nephrotic syndrome in Iran: a single center study. Indian J Nephrol. 2009;19(3):87-90. doi:10.4103/0971-4065.57103

2. Ishikura K, Matsumoto S, Sako M, et al. Clinical practice guideline for pediatric idiopathic nephrotic syndrome, medical therapy. Clin Exp Nephrol. 2015;19(1):6-33. doi:10.1007/s10157-014-1030-x

3. Chaubey S, Singh KV, Singh P, Mittal M, Singh Abhishek K, Kushwaha KP. A study on intima-media thickness of carotid artery in children with nephrotic syndrome: a cross sectional study. $J$ Pediatr Rev. 2017;4(02):91-101.

4. Zhang Q, Zeng C, Fu Y, Cheng Z, Zhang J, Liu Z. Biomarkers of endothelial dysfunction in patients with primary focal segmental glomerulosclerosis. Nephrology. 2012;17(4):338-345. doi:10.1111/ j.1440-1797.2012.01575.x

5. Szeto CC, Chow KM, Woo KS, et al. Carotid intima media thickness predicts cardiovascular diseases in Chinese predialysis patients with chronic kidney disease. J Am Soc Nephrol. 2007;18(6):1966-1972. doi:10.1681/ASN.2006101184

6. Touboul P-J, Hennerici MG, Meairs S, et al. Mannheim carotid intima-media thickness and plaque consensus (2004-2006-2011). An update on behalf of the advisory board of the 3rd, 4th and 5th watching the risk symposia, at the 13th, 15th and 20th European Stroke Conferences, Mannheim, Germany, 2004, Brussels, Belgium, 2006, and Hamburg, Germany, 2011. Cerebrovasc Dis. 2012;34 (4):290-296. doi:10.1159/000343145 
7. Srivastava RN, Bagga A. Nephrotic Syndrome. ch. 12. 4th. New Delhi, India: Pediatric Nephrology, Jaypee Brothers Medical Publishers (P) Ltd.; 2005:161-197.

8. Adhikari MC, Guin A, Chakraborty S, et al. Subclinical atherosclerosis and endothelial dysfunction in patients with early rheumatoid arthritis as evidenced by measurement of carotid intima-media thickness and flow-mediated vasodilatation: an observational study. Semin Arthritis Rheum. 2012;5:669-675. doi:10.1016/j.semarthrit.2011.08.003

9. Kniazewska MH, Obuchowicz AK, Wielkoszyn'Oski T, et al. Atherosclerosis risk factors in young patients formerly treated for idiopathic nephrotic syndrome. Pediatr Nephrol. 2009;24:549-554. doi:10.1007/s00467-008-1029-1

10. Kuźma-Mroczkowska E, Pańczyk-Tomaszewska M, Skrzypczyk P, et al. Body weight changes in children with idiopathic nephrotic syndrome. Dev Period Med. 2016;20:16-22.

11. El-Mashad GM, El-Hawy MA, El-Hefnawy SM, et al. Bone mineral density in children with idiopathic nephrotic syndrome. $J$ Pediatr (Rio J). 2016;16:237-266.

12. Rahul I, Krishnamurthy S, Satheesh S, et al. Brachial artery flow-mediated dilatation and carotid intima medial thickness in pediatric nephrotic syndrome: a cross-sectional case-control study. Clin Exp Nephrol. 2015;19:125-132. doi:10.1007/s10157-014-0958-1

13. Youssef DM, Gomaa MA, El-Akhras A, et al. Brachial artery flow-mediated dilatation and carotid intima-media thickness in children with idiopathic nephrotic syndrome. IJKD. 2018;12:331-340.

14. Nakamura A, Niimi R, Kurosaki K, et al. Factors influencing cardiovascular risk following termination of glucocorticoid therapy for nephrotic syndrome. Clin Exp Nephrol. 2010;14(5):457-462. doi:10.1007/s10157-010-0317-9

15. Candan C, Canpolat N, Gökalp S, et al. Subclinical cardiovascular disease and its association with risk factors in children with steroid-resistant nephrotic syndrome. Pediatr Nephrol. 2014;29 (1):95-102. doi:10.1007/s00467-013-2608-3

16. Valavi E, Aminzadeh M, Amouri P, Rezazadeh A, Mousavi MB. Effect of prednisolone on linear growth in children with nephrotic syndrome. J De Pediatria (Versão Em Português). 2020;96 (1):117-124. doi:10.1016/j.jpedp.2018.11.019

17. Pritchard KA, Groszek L, Smalley DM, et al. Native low-density lipoprotein increases endothelial cell nitric oxide synthase generation of superoxide anion. Circ Res. 1995;77(3):510-518. doi:10.1161/01. RES.77.3.510
18. Dogra GK, Herrmann $\mathrm{S}$, Irish $\mathrm{AB}$, et al. Insulin resistance, dyslipidaemia, inflammation and endothelial function in nephrotic syndrome. Nephrol Dial Transplant. 2002;17:2220-2225. doi:10. 1093/ndt/17.12.2220

19. Tkaczyk M, Czupryniak A, Owczarek D, et al. Markers of endothelial dysfunction in children with idiopathic nephrotic syndrome. $\mathrm{Am}$ J Nephrol. 2008;28(2):197-202. doi:10.1159/000110088

20. Hooman N, Isa-Tafreshi R, Otukesh H, Mostafavi SH, Hallaji F. Carotid artery function in children with idiopathic nephrotic syndrome. Nefrologia. 2013;33:650-656. doi:10.3265/Nefrologia. pre2013.May.12036

21. Mehta A, Mishra S, Ahmad K, Tiwari HC, Singh V, Singh A. Carotid intima media thickness in children with nephrotic syndrome: an observational case control study. Sudan J Paediatr. 2019;19 (2):110-116. doi:10.24911/SJP.106-1535804613

22. Dalmau SJ, Vitoria MI, Legarda TM, Muro VD, Sangüesa NC. Evaluation of carotid intima-media thickness in familial hypercholesterolemia in childhood. An Pediatr. 2009;70(4):349-353. doi:10. 1016/j.anpedi.2008.11.017

23. Mitsnefes M, Flynn J, Cohn S, et al. CKD Study Group masked hypertension associates with left ventricular hypertrophy in children with CKD. J Am Soc Nephrol. 2010;21(1):137-144. doi:10.1681/ ASN.2009060609

24. Frishberg Y, Feinstein S, Rinat C, et al. The heart of children with steroid-resistant nephrotic syndrome: is it all podocin? J Am Soc Nephrol. 2006;17(1):227-231. doi:10.1681/ASN.2005060653

25. Giuseppe S, Gaetano V, Gianpaolo R, et al. High-density lipoprotein cholesterol and left ventricular hypertrophy in essential hypertension. J Hypertens. 2001;19(12):2265-2270. doi:10.1097/00004872-200112 000-00021

26. Birjmohun RS, van Leuven SI, Levels JHM, et al. High-density lipoprotein attenuates inflammation and coagulation response on endotoxin challenge in humans. Arterioscler Thromb Vasc Biol. 2007;27:1153-1158. doi:10.1161/ATVBAHA.106.136325

27. Vaziri ND, Liang K, Parks JS. Acquired lecithin-cholesterol acyltransferase deficiency in nephrotic syndrome. Am J Physiol $R$ En Physiol. 2001;28:F823-F828. doi:10.1152/ajprenal.2001.280.5.F823
Vascular Health and Risk Management

\section{Publish your work in this journal}

Vascular Health and Risk Management is an international, peerreviewed journal of therapeutics and risk management, focusing on concise rapid reporting of clinical studies on the processes involved in the maintenance of vascular health; the monitoring, prevention and treatment of vascular disease and its sequelae; and the involvement of metabolic disorders, particularly diabetes. This journal is indexed on PubMed Central and MedLine. The manuscript management system is completely online and includes a very quick and fair peerreview system, which is all easy to use. Visit http://www.dovepress. com/testimonials.php to read real quotes from published authors. 